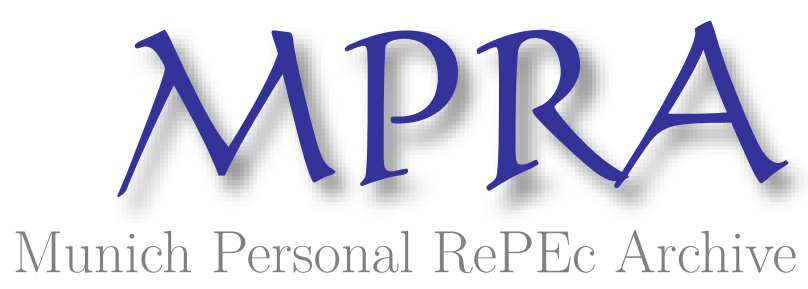

\title{
A propensity score analysis of public incentives: The Italian case
}

Affuso, Antonio

2011

Online at https://mpra.ub.uni-muenchen.de/36698/

MPRA Paper No. 36698, posted 17 Feb 2012 10:44 UTC 


\title{
A propensity score analysis of public incentives: The Italian case.
}

\author{
Antonio Affuso
}

a.affuso@gmail.com

Keywords: Propensity score; Credit rationing; Public subsidies

JEL: $\mathrm{C} 2, \mathrm{H} 2$

\begin{abstract}
Public support to firms has been a traditional and important industrial policy measure in many countries for several decades. One of the reasons for public intervention is the existence of market failures or imperfections. Informational asymmetries between borrowers and lenders of funds in particular are used to justify subsidies to firms, especially small and medium-sized enterprises. Within this framework, the main purpose of public subsidies is offsetting market imperfections.

This paper makes a contribution to current empirical literature by examining the effects of public funding on credit rationing of small and medium-sized Italian firms.

The results suggest that public subsidies reduce the probability of a firm being credit rationing.
\end{abstract}




\section{Introduction}

Public support to firms has been a traditional and important industrial policy measure in many countries for several decades. One of the reasons for public intervention is the existence of market failures or imperfections. Informational asymmetries between borrowers and lenders of funds in particular are used to justify subsidies to firms, especially small and medium-sized enterprises. Within this framework, the main purpose of public subsidies is offsetting market imperfections. Although there is a great deal of literature on the effect of state aid in Italy, there is no agreement on its effectiveness. See Bagella and Becchetti (1998), Bronzini and De Blasio (2006) and Adorno, Bernini and Pellegrini (2007). These papers and many others focus on the effects on productivity, debt ratio, profitability and employment, but no empirical studies so far have analyzed the impact of public subsidies on credit rationing.

This paper therefore makes a contribution to current empirical literature by examining the effects of public funding on credit rationing of small and medium-sized Italian firms.

The basic idea of the paper is that public subsidies affect firms' ability to obtain more funds. This is because incentive has a positive effect on investments, which, in turn, act negatively on credit rationing through collateralization.

The problem of self-selection arises in this analysis, because in public financing programs, firms are selected on the basis of common characteristics. So subsidized firms and unsubsidized firms cannot be considered random draws. In order to overcome this problem, I use a Propensity Score Matching model.

The paper is organized as follows. Section 2 describes methodological issues and presents the database, Section 3 summarises the main results, and the last Section concludes.

\section{Methodological issues}

I focus on propensity score matching model to investigate a causal link between public incentives and firms' rationing.

Matching has become a popular approach to estimate casual treatment effects and empirical examples can be found in very different fields of study. Since they were introduced by Rosenbaum and Rubin (1983), propensity scores have been used in observational studies in many fields. Propensity score methods are relatively new to the economic literature; recent applications include Dehejia and Wahba (1999), Heckman, Ichimura, and Todd (1997), and Lechner (1999).

In this section I focus on propensity score matching model (Rosenbaum and Rubin (1983)) to investigate a causal link between public incentives and firms' rationing.

Matching methods, of which propensity score matching is an important subset, are attractive because focus attention on a specific causal effect of interest, and treat all variables other than the treatment variable as potentially confounding variables. In the matching approach, the influence of confounding variables is reduced by matching the potentially confounding covariates of the cases that experienced the treatment with cases that did not experience the treatment. However, the underlying identification requirement is that the program choice is independent of outcomes conditional on certain set of observables.

This assumption would be violated if unobserved characteristics of the individual independently impacted the likelihood of receiving treatment. The most common strategy for dealing with this problem is the use of instrumental variables (IV) estimator. Indeed, instrumental variables estimator provides an alternative strategy for the estimation of causal effects. Instrumental variables approach involves identifying instruments that are related to treatment but not to outcomes other than through their effects on treatment. 
Imbens and Angrist (1994), however, have shown that the IV estimator for the treatment effect applies only under the unrealistic case where the treatment effect is constant within the population. In the more general case when responses to treatment vary among persons with the same characteristics, the method of instrumental variables breaks down without special assumptions.

Heckman (1997) shows that if responses to treatment vary, and if we are interested in estimating the mean effect of treatment on the treated, any valid application of the method of instrumental variables for estimating the treatment effects requires a behavioral assumption about how persons make their decisions about program participation.

Under a set of additional assumptions the IV estimator estimates the average effect of treatment for the subsample (LATE) of the population that is induced by a specific change in the value of the IV to select themselves into treatment. These assumptions introduce new sources of uncertainty. Furthermore, the LATE estimator relies for its consistency on the assumptions that the assignment to treatment mechanism is ignorable. So, instrumental variables estimation can eliminate endogeneity bias under a set of assumptions that themselves are rather strong and impractical to verify in most real research setting.

Moreover, Ichimura and Taber (2001) show that conditions justifying instrumental variables methods justify the matching method as a special case. With this in mind, in this paper I use propensity score method for its explanatory power and because it allows to appreciate immediately the equivalence of treatment and control groups and to perform simple matched pair analyses which adjust for confounding variables.

The propensity score is the probability for an individual of participating in a treatment given his observed covariates $X$. In this methodology treatment assignment and potential outcome are independent on propensity score.

In a sample of size $N$, for each individual $i$ in the sample, for $i=1 \ldots N$, let $D_{i}$ indicate whether the treatment was received, with $D_{i}=1$ if individual $i$ receives the treatment, and $D_{i}=0$ if individual $i$ does not receive the treatment. Let $\left(Y_{i}(0), Y_{i}(1)\right)$ denote the two potential outcomes, $Y_{i}(0)$ is the outcome of individual $i$ when it is not exposed to the treatment and $Y_{i}(1)$ is the outcome of individual $i$ when it is exposed to the treatment. If both $Y_{i}(1)$ and $Y_{i}(0)$ were observable, then the effect of the treatment on $i$ would be $Y_{i}(1)-Y_{i}(0)$.

However, only one of the two potential outcomes is observed for each individual and the other is unobserved or missing.

The causal effect $\theta$ that results from receiving the treatment is:

$$
\theta=E[Y(1)-Y(0) \mid D=1]=E[Y(1) \mid D=1]-E[Y(0) \mid D=1]
$$

where $D=(0,1)$ is the indicator of exposure to the treatment. However, the counterfactual mean for those being treated, $E[Y(0) \mid D=1]$, is not observable and in non-experimental studies it cannot be calculated as the arithmetic mean of non-treated units since:

$$
E[Y(0) \mid D=1] \neq E[Y(0) \mid D=0]
$$

Taking the mean outcome of non-treated individuals is not advisable since treated and non-treated individuals differ also in the absence of treatment. In non-experimental studies identifying assumptions need to be made to solve this problem.

Rubin (1977) introduces the Conditional Independence Assumption (CIA). The CIA means that participation and potential outcome are independent for individuals with the same set of exogenous characteristics $\left(X=x_{i}\right)$ : 
If CIA holds, $E\left[Y(0) \mid D=0, X=x_{i}\right]$ can be used as a measure of potential outcome. CIA, however, is only valid if all variables that influence treatment assignment and potential outcomes are observed simultaneously. This is a strong assumption that also requires a large number of exogenous characteristics.

Rosenbaum and Rubin (1983) suggest using "balancing scores" to reduce the vector of exogenous variables into a single scalar measure. They show that if potential outcomes are independent of treatment conditional on $X$, they are also independent of treatment conditional on a balancing score $b(X)$. The propensity score $p(D=1 \mid X)=p(X)$ is one possible balancing score. Rosenbaum and Rubin proposed propensity score matching as a method of reducing the bias in the estimation of treatment effects with observational data sets. For each individual, other individuals whose characteristics are similar to those being treated, but who were not exposed to the treatment, are used to calculate the counterfactual. The propensity score is used to select from the control group the most comparable counterpart. This corrects for selection bias that stems from differences between the two groups.

The propensity score satisfies two important properties that reduce bias in the estimation procedure:

- Balancing property: $D \perp X \mid p(X)$; ensures that, given the propensity score, the treatment and the observables are independent;

- Unconfoundedness property: if $(Y(1), Y(0)) \perp D \mid X$ then $(Y(1), Y(0)) \perp D \mid p(X)$; ensures that, given the propensity score, the treatment and potential outcomes are independent.

The difficulty with matching estimators lies in determining when matches for treated and nontreated individuals are close enough.

The methodology I use was developed by Becker and Ichino (2002). The method is based on the computation of the propensity score using a standard probit or logit model. Using the predicted propensity score for each individual, the sample is divided into equally-spaced intervals of the propensity score. Within each block, the mean propensity scores for treated and non-treated individuals are compared and tested to see if they are identical. If they are not, the interval is split in half. Once equality of propensity score has been achieved, characteristics of individuals within blocks are compared to see if they are identical on average. If this balance is achieved within blocks, the average effect of treatment on treated (ATT) is calculated.

In calculating the ATT, various methods have been proposed to overcome the problem that the probability of observing two individuals with exactly the same value of the propensity score is in principle zero since $p(X)$ is a continuous variable. In this paper I use the Nearest Neighbor Matching method.

In the Nearest Neighbor Matching estimator, the individual from the comparison group is chosen as a matching partner for a treated individual that is closest in terms of propensity score. It uses a single match and hence ensures the smallest propensity-score distance between the two units.

Becker and Ichino (2002) underline that in the Nearest Neighbor method, all treated units find a match. However, it is obvious that some of these matches are fairly poor, because for some treated units the Nearest Neighbor may have a very different propensity score and nevertheless still contribute to the estimation of the treatment effect.

The data used in this paper come from the Survey of Manufacturing Firms carried out by Area Studi of Capitalia Bank. I use two samples, one for the period 1995-1997, and one for the period 19982000. Data are available until 2006 but I did not use this survey because it would reduce significantly the number of observations. Indeed, many firms which are in the period 1995-2000 are missing in the following period. 
The samples were stratified according to size, industry, and location and thus constitute a statistically significant representation of Italian manufacturing industry.

The database contains microdata at firm level for Italian enterprises and contains balance sheet information as well as information about direct public financial subsidies and credit rationing. In this section I refer to a panel which comprises the subsample of firms that are always present during the time window. In order to identify the firms having received public aids I use the information from the survey covering 1995-1997. Indeed this information is given at the end of the period and is referred to the previous three years. In this way, using data on credit rationing from the survey years 1998-2000, I can observe the effect of receiving public incentives in the second period.

I assume that a firm is credit rationed if it answers 'Yes' to two of these three questions on access to credit:

(i) Whether at the current market interest rate they wish a larger amount of credit; (ii) Whether they would be willing to accept a small increase in the interest rate charged in order to obtain more credit; (iii) Whether they have applied for credit but have been turned down by the financial intermediary.

I define outcome $Y_{i}$ for firm $i$ from a sample of $i=1 \ldots . N$ firms. I assume that $Y_{i}$ is binary and represents whether or not a firm is rationed. The observed covariates $X$ which are used in this analysis are the share of fixed assets on total assets as a proxy for asset collateral value (Johnson, 1997). In fact, tangibility eases the availability of debt and improves the terms on which debt is available. The collateral value of fixed assets depends directly on the liquidation value, so it is possible to use this measure as a proxy for project liquidation values. Because leverage is positively associated with liquidation value (Harris and Raviv 1990), liquidation value is negatively linked with credit rationing; the share of long term financial assets on total assets; the ratio between short term financial assets and total assets; the ratio of gross operating surplus on total sales as a measure of profitability; the productivity is measured by the ratio of sales per worker; and the total bank debt of the firm divided by total liabilities. There are also a dummy variable that represents the firm size.

$D$ represents subsidies to firms; $D=1$ if the firm receives subsidy, $D=0$ otherwise. The problem of missing data arises because for each individual only one outcome is observed, that the firm is subsidized or not, but never both. The decision of a firm to apply for public assistance as well as the selection mechanisms generate a group of firms with special characteristics. In fact firms' characteristics influence the probability of receiving subsidies. A comparison between firms using the initial data set would therefore lead to biased results due to the difference between both groups.

In my panel some covariates have missing data. To estimate propensity scores I use a complete-data analysis which uses only observations where all variables are observed.

The hypothesis is that missing values are missing completely at random (MCAR), in other words missing values are randomly distributed throughout the panel. If the missing values are MCAR, then the listwise deletion will give unbiased estimates. The only disadvantage is a reduction in statistical power, but this is not a problem if the sample is sufficiently large.

I use the propensity score matching approach to investigate the effects of public subsidies on firms' credit rationing. Credit rationing can thus be thought of as a proxy of a firm's performances, in the same way as firm's productivity and profitability.

\section{Results}

In this Section I present the main results of the analysis. They show that direct subsidies significantly lower the level of firms' rationing. This confirms the interpretation of some literature which considers public intervention as an opportunity to correct market failures. 
I estimate the propensity score using the procedure developed by Becker and Ichino (2002). In the first step, the procedure identifies the optimal number of blocks that ensures that the mean propensity score is not different for treated units and controls in each blocks. In my case the final number of blocks is 9 .

In the second step, the balancing property of the propensity score is tested and is satisfied in my analysis.

I impose the common support condition, which implies that the test is performed only on the observations whose propensity score lies in the intersection of the supports of the propensity score of treated and controls, to improve the quality of the matches used to estimate the ATT. To estimate ATT, I use the Nearest Neighbor Matching method.

As the Table below shows, the Nearest Neighbor method shows that the effect of public subsidies on the ATT is always negative and significantly different from zero. This means that subsidized firms are less credit rationed than non-subsidized firms.

More specifically, the likelihood of being rationed is reduced by 2.6 percentage points.

Table 1: ATT Estimates

\begin{tabular}{|l|l|}
\hline & Nearest Neighbor \\
\hline Estimate (ATT) & -0.026 \\
t-value & -1.945 \\
\hline n. treated & 504 \\
n.controls & 291 \\
\hline
\end{tabular}




\section{Conclusions}

I analyze the effects of public incentives on credit rationing. I use the propensity score method to avoid self-selection problems and I find that public subsidies reduce the probability of a firm being credit rationing.

Is possible to interpret these findings in different ways. On the one hand, public subsidies increase the amount of fixed investments and allow firms to have more collateral to offer to the banks. Public subsidies in fact change the firm's capital structure. Bagella and Becchetti (1998) partially confirm this hypothesis; they find that in the short run, subsidies cause a higher level of indebtedness. Moreover, the public screening process can be considered by the banks as a preliminary selection process of firms. Firms that receive subsidies have safer investment projects. Third, because investment in machinery and equipment has a positive effect on productivity, as shown by De Long and Summers (1991), investment subsidies may increase firms' productivity in the short run, diminishing the credit rationing of firms. 
Bibliography

Adorno, V., C. Bernini and Pellegrini, G. (2007), "The Impact of Capital Subsidies: New Estimations Under Continuous Treatment", Giornale degli Economisti e Annali di Economia, 66, 1.

Bagella, M. and Becchetti, L. (1998), "Gli Effetti di Breve Periodo delle Agevolazioni: il Costo del Debito, l'Offerta di Credito, gli Investimenti e l'Effetto del Ciclo”. In: M.Bagella, (ed.), Gli Incentivi di Politica Industriale: Presupposti Teorici e Valutazioni Empiriche, 34-100. [In Italian].

Becker, S.O. and Ichino, A. (2002), "Estimation of average treatment effects based on propensity scores", The Stata Journal, 2(4), 358-377.

Bronzini, R. and De Blasio, G. (2006), "Evaluating the impact of investment incentives: The case of Italy's Law 488/1992", Journal of Urban Economics 60, 327-349.

Dehejia, R., and Wahba, S. (1999), "Causal Effects in Non-Experimental Studies: Re-Evaluating the Evaluation of Training Programs", Journal of the American Statistical Association, 94, 1999, 1053-1062

DeLong, J.B., and Summers, L.H. (1991), "Equipment Investment and Economic Growth", Quarterly Journal of Economics, 106(2): 445-502.

Harris, M., and Raviv, A. (1991), "The Theory of Capital Structure", The Journal of Finance, 46(1): 297-355.

Heckman, J. (1997), "Instrumental Variables: A Study of Implicit Behavioral Assumptions Used in Making Program Evaluations" The Journal of Human Resources, Vol. 32, No. 3, pp. 441-462

Heckman, J., Ichimura, H. and Todd, P. (1997), "Matching as an Econometric Evaluation Estimator: Evidence from Evaluating a Job Training Program", Review of Economic Studies, 64, 605-654

Ichimura, H., and Taber, C. (2001), "Propensity-Score Matching with Instrumental Variables" The American Economic Review, Vol. 91, No. 2, Papers and Proceedings of the Hundred Thirteenth Annual Meeting of the American Economic Association, pp. 119-124

Imbens G., and Angrist, J. (1994), "Identification and Estimation of Local Average Treatment Effects Published", Econometrica, Vol. 62, No. 2, 467-476

Johnson, S.A. (1997), "An Empirical Analysis of the Determinants of Corporate Debt Ownership Structure", The Journal of Finance and Quantitative Analysis, 32(1): 47-69.

Lechner, M., (1999), "Earnings and Employment Effects of Continuous Off-the-job Training in East Germany after Unification”, Journal of Business and Economic Statistics, 17, 1999, 74-90

Rosenbaum P.R., and Rubin, D.B. (1983), "The Central Role of the Propensity Score in Observational Studies for Causal Effects", Biometrika, Vol. 70, No. 1., 41-55.

Rubin D.B., (1977), "Assignment to Treatment Group on the Basis of a Covariate", Journal of Educational Statistics, Vol. 2, No. 1, 1-26. 